\title{
Expression stability of putative reference genes in equine endometrial, testicular, and conceptus tissues
}

\author{
Claudia Klein ${ }^{1 *}$, Josep Rutllant ${ }^{2}$ and Mats HT Troedsson ${ }^{1}$
}

\begin{abstract}
Background: Quantitative RT-PCR data are commonly normalized using a reference gene. A reference gene is a transcript which expression does not differ in the tissue of interest independent of the experimental condition. The objective of this study was to evaluate the stability of mRNA expression levels of putative reference genes in three different types of equine tissue, endometrial, testicular, and conceptus tissue.

Findings: The expression stability of four (uterine tissue) and six (testicular and conceptus tissue) was assessed using descriptive data analysis and the software programs Normfinder and geNorm. In uterine samples, 185 showed the largest degree of variation in expression while GAPDH, B2M, and ACTB were stably expressed. B2M and GAPDH were identified as the most stably expressed genes in testicular samples, while 185 showed some extent of regulation between samples. Conceptus tissue overall was characterized by very low variability of the transcripts analyzed with GAPDH, YWHZ, and $18 \mathrm{~S}$ being the most stably expressed genes.

Conclusions: In equine endometrium, GAPDH, B2M, and ACTB transcript levels are equally stable, while 185 is less stably expressed. In testes and associated structures, B2M and GAPDH are the transcripts showing the least amount of variation, while in conceptus tissue GAPDH, YWHZ, and 185 were identified as the most suitable reference genes. Overall, transcripts analyzed in conceptus tissue were characterized by less variation than transcripts analyzed in uterine and testicular tissue.
\end{abstract}

\section{Background}

Real-time reverse transcription PCR (Real-time RT-PCR), also referred to as quantitative PCR (RT-qPCR) is a powerful tool to determine quantitative changes in mRNA expression levels and is widely applied in reproductive biology research. This technology provides a means to compare the abundance of a certain transcript of interest in tissue obtained from different biological statuses or to assess the impact of an experimental treatment on the expression of the gene of interest. Normalization procedures are an essential step in the analysis of real-time RT-PCR data to ensure that observed changes in transcript abundance reflect biological variation rather than systematic variation. Sources for systematic or non-biological variation include the

\footnotetext{
* Correspondence: claudia.klein@uky.edu

'University of Kentucky, Department of Veterinary Science, 108 Gluck Equine Research Center, Lexington, KY, 40546, USA

Full list of author information is available at the end of the article
}

amount of RNA input into the reverse transcription reaction, the efficiency of the reverse transcription reaction, pipetting accuracy while preparing the PCR reaction, and the presence of PCR inhibitory substances in a sample. Normalization procedures are therefore applied to correct for the occurrence of these systematic variations. A common normalization procedure is to report the expression level of the transcript of interest relative to the expression level of a reference gene. A reference gene, also referred to as maintenance or internal control gene, is a transcript whose expression level is constant in the specific tissue of interest, i.e. its mRNA abundance does not change with experimental treatment or with different biological conditions [1]. Stability of the reference gene/genes used in real-time RT-PCR events is crucial to avoid changes in gene expression of the transcript of interest due to their variation being interpreted as biological variation. Studies to determine reliable reference genes for equine skin and equine lymphocytes during
C Biomed Central

(c) 2011 Klein et al; licensee BioMed Central Ltd. This is an open access article distributed under the terms of the Creative Commons Attribution License (http://creativecommons.org/licenses/by/2.0), which permits unrestricted use, distribution, and reproduction in any medium, provided the original work is properly cited. 
exercise induced stress have been carried out $[2,3]$. There is no consensus regarding the most appropriate gene to use as an internal reference gene in RT-qPCR utilizing equine endometrial, testicular, or conceptus tissue samples. The objective of this study was to evaluate the stability of mRNA expression levels of putative reference genes in three different types of equine tissue, endometrial, testicular, and conceptus tissue.

\section{Materials and methods \\ Animals and Tissue Collection \\ Collection of endometrium and conceptuses}

All animal procedures were completed in accordance with and with the approval of the Institutional Animal Care and Use Committee at the University of Kentucky (protocol number 2008-0351). Endometrial tissue samples were recovered from cyclic mares 8 days $(n=4), 10$ days $(n=5)$, 12 days $(n=6), 14$ days $(n=5)$, and 16 days $(n=4)$ after ovulation, from pregnant mares endometrial tissue samples were recovered at Day $10(n=4)$, Day $12(n=4)$, and Day $16(n=3)$ of pregnancy. Pregnancy status was confirmed via transrectal ultrasonography. If no embryo was detected the mare was excluded from sample collection. Endometrial tissue samples were recovered transcervically using a punch biopsy instrument. Tissue specimens were snap-frozen in liquid nitrogen and stored at $-80^{\circ} \mathrm{C}$ till further processing. Tissue samples were collected from a total of 17 different mares. Ten mares were used more than once for sample collection such that a total of 35 samples could be collected. Conceptuses were retrieved from pregnant mares through transcervical flush. Four conceptuses each were recovered $8,10,12$, and 14 after ovulation.

\section{Collection of testis and associated structures}

Testes and associated structures were collected from mature stallions during routine castration and transported on ice to the lab within $30 \mathrm{~min}$. Samples were obtained from testicular parenchyma $(\mathrm{n}=11)$, head $(\mathrm{n}=$ $4)$, body $(n=4)$, and tail of the epididymis $(n=5)$, and the ductus deferens $(n=6)$. Tissue specimens were snap-frozen in liquid nitrogen and stored at $-80^{\circ} \mathrm{C}$ till further processing.

\section{Isolation of RNA}

Total cellular RNA from endometrial and testicular samples was isolated using Trizol reagent (Invitrogen, Carlsbad, CA) according to the manufacturer's recommendation followed by precipitation using an equal volume of isopropanol and 1/10 volume of $3 \mathrm{M}$ sodium acetate. Total cellular RNA from conceptus tissue was isolated using RNeasy Mini kit (Quiagen, Valencia, CA). RNA was quantified via spectrophotometry using a NanoDrop ND-1000. Samples with a 260/280 ratio of 1.95 or greater and a $260 / 230$ ratio of 2.0 or greater were used for analysis.

\section{Real-time RT-PCR}

Real-time RT-PCR experiments were carried out under the consideration of the MIQE guidelines [4]. RNA samples $(2 \mu \mathrm{g} /$ reaction for endometrial and testicular specimens, and $200 \mathrm{ng} /$ reaction for conceptus specimens) were treated with RNase-free DNase I (Ambion, Austin, $\mathrm{TX})$ for $15 \mathrm{~min}$ at $37^{\circ} \mathrm{C}$, heat denatured $\left(75^{\circ} \mathrm{C}\right.$ for 10 min), then reverse transcribed using High Capacity cDNA Reverse Transcription Kit and random hexamers (Applied Biosystems, Foster City, CA). cDNA was purified using the QIAquick ${ }^{\circledR}$ PCR Purification Kit (Qiagen, Germantown, MD) and cDNA concentration was determined via spectrophotometry. Purified cDNA (50 ng) was used for each PCR reaction.

For endometrial specimen, the mRNA expression of four putative reference genes, glyceraldehyde 3-phosphate dehydrogenase (GAPDHP), 18S rRNA (18S), beta2-microglobulin $(B 2 M)$, and beta actin $(A C T B)$ and one non-reference gene, solute carrier family 36 member 2 (SLC36A2) were measured by real-time RT-PCR. For testicular samples, the mRNA expression of GAPDH, $18 S, B 2 M, A C T B$, Succinate dehydrogenase complex $(S D H A)$, and beta glucoronidase (GUSB) as putative internal control genes and aromatase (Cyp19a1) as nonreference transcript were determined. For conceptus tissue, the expression of $G A P D H, 18 S, B 2 M, A C T B$, $S D H A$, and tyrosine 3-monooxygenase/tryptophan 5monooxygenase activation protein (YWHAZ) as reference gene candidates and Cyp19a1as non-reference genes was assessed using quantitative PCR. Primers specific for the selected transcripts were designed using Jellyfish 3.3.1 (Field Scientific LLC, Lewisburg, PA) and are listed in Table 1. Specificity of the primers was confirmed via sequencing of the PCR products to confirm amplification of the intended target sequence. Primer efficiency was assessed using Linreg http://www.genequantification.de to ensure all primers resulted in PCR efficiencies of at least 1.9. Real-time PCR was completed using SYBR Green PCR Master Mix (Applied Biosystems) with the following cycling conditions: $95^{\circ} \mathrm{C}$ for 10 min; 40 cycles of $95^{\circ} \mathrm{C}$ for $15 \mathrm{sec}, 59^{\circ} \mathrm{C}$ for $1 \mathrm{~min} ; 55$ to $95^{\circ} \mathrm{C}$ for dissociation. Each PCR was performed in triplicate. Specificity of amplification was monitored by including non-reverse transcribed RNA reactions for each sample and by completing a dissociation analysis at the end of each real-time run to verify the amplification of a single product. Cycle threshold $(\mathrm{Ct})$ values were obtained through the auto $\mathrm{Ct}$ function.

\section{Data analysis}

Data were analyzed separately but in the same fashion for endometrial, testicular, and conceptus tissue. $\mathrm{Ct}$ values from the ABI PRISM 7000 Sequence Detection System (Applied Biosystems) were exported into Excel 
Table 1 Primer sequences used in the current study

\begin{tabular}{|c|c|c|c|c|}
\hline Gene & GenBank accession \# & Forward primer ( $5^{\prime}$ to $\left.3^{\prime}\right)$ & Reverse primer ( $3^{\prime}$ to $\left.5^{\prime}\right)$ & product size \\
\hline GUSB & XM_001493514 & GGGATTCGCACTGTGGCTGTCA & CCAGTCAAAGCCCTTCCCTCGGA & 116 \\
\hline Cyp19a1 & NM_001081805 & GAGATGCCGTGGGAATTCTA & CCACGTTTCTCAGCCAAAAT & 111 \\
\hline$A C T B$ & NM_001081838 & CGACATCCGTAAGGACCTGT & CAGGGCTGTGATCTCCTTCT & 99 \\
\hline$B 2 M$ & NM_001082502 & GTGTTCCGAAGGTTCAGGTT & ATTTCAATCTCAGGCGGATG & 102 \\
\hline SLC36A2 & XM_001501324 & GCTTCTGCCACAGGCTTAAC & CCGGCTTTGAGTCCATACAT & 69 \\
\hline GAPDH & NM_001163856 & AGAAGGAGAAAGGCCCTCAG & GGAAACTGTGGAGGTCAGGA & 87 \\
\hline SDHA & XM_001490889 & GCAGAAGAAGCCATTTGAGG & CCTGTCGATTACGGGTCTGT & 103 \\
\hline YHWAZ & XM_001492988 & TGTTGTAGGAGCCCGTAGGT & ATTCTCGAGCCATCTGCTGT & 95 \\
\hline 185 & XM_001497064 & AACGACACTCTGGCATGCTAACTA & CGCCACTTGTCCCTCTAAGAA & 98 \\
\hline
\end{tabular}

(Microsoft Corporation, Redmond, WA). Excel and GraphPadPrism (La Jolla, CA) were used for further analysis of the dataset. Linreg was used to determine the efficiencies of each PCR reaction. Efficiency corrected Ct values from triplicate reactions were averaged for further analysis and are from now on referred to as $\mathrm{Ct}$ value. Mean Ct values, their standard error of measurement (SEM) and their coefficient of variation across the 35 samples were calculated for each of the putative reference genes. Ct values were logarithmically transformed and mean log transformed $\mathrm{Ct}$ values for each gene visualized using box and whisker plots. For each sample, ratios of mean $\mathrm{Ct}$ values among the putative reference genes were formed including all possible combinations. The mean and the coefficient of variation $(\mathrm{CV})$ of the resulting ratios were calculated. Box and whisker plots were used to visualize the ratios of mean $\mathrm{Ct}$ values.

Two freely available software packages designed to identify stably expressed genes among a set of candidate genes, NormFinder http://www.mdl.dk/publicationsnormfinder.htm and geNorm http://medgen.ugent.be/ jvdesomp/genorm/, were used for further expression stability analysis. Bestkeeper [5] is another freely available software that determines stable reference genes through pair-wise correlation analysis. This software was not used in the current study to limit the extent of the manuscript.

\section{NormFinder}

NormFinder is publicly available software that uses an algorithm to identify the optimal normalization gene among a set of candidates. It ranks the set of candidate normalization genes according to their expression stability in a given sample set. The output provided by NormFinder includes an arbitrary stability value for each gene. The model and statistical framework underlying this software are described in Andersen et al. [6].

geNorm

geNorm is publicly available software package that determines the most stable genes from a set of tested genes. This application differs from NormFinder in that it determines the stability of the best pair of reference genes and not the most stable individual gene. The underlying principles and calculations are described in Vandesompele et al. [7].

\section{Calculation of delta values}

Delta Ct $(\triangle \mathrm{Ct})$ values for SLC36A2 (endometrial samples), and Cyp19a1 (testicular and conceptus tissue) were calculated using each of the putative reference genes. Delta delta $\mathrm{Ct}(\Delta \Delta \mathrm{Ct})$ values relative to expression levels at Day 8 of the cycle (endometrial samples), ductus deferens (testicular samples), and Day 8 conceptus (conceptus tissue) respectively were then determined. Pearson's correlation coefficients were calculated for the resulting $\Delta \Delta \mathrm{Ct}$ values using SAS (SAS, Cory, $\mathrm{NY}$ ).

\section{Results}

\section{Endometrial samples}

$18 S$ showed the highest level of mRNA abundance with a mean Ct value of $9.01 \pm 0.25$, whereas GAPDH, $A C T B$, and $B 2 M$ mRNA showed lower expression levels with mean $\mathrm{Ct}$ values of $23.47 \pm .21,21.29 \pm 0.23$, and $20.64 \pm 0.24$, respectively.

Mean Ct values for $18 \mathrm{~S}$ displayed the greatest amount of deviation with a CV of $17.07 \%$. Mean $\mathrm{Ct}$ values for GAPDH displayed the least amount of variation with a $\mathrm{CV}$ of $5.24 \%$. Mean $\mathrm{Ct}$ values for $A C T B$ and $B 2 M$ showed a similar degree of variability as those for GAPDH, with coefficient of variations of $6.39 \%$ and $7.04 \%$ (Table 2). Figure 1 displays the log transformed mean cycle threshold values for each of the four putative reference genes in the form of box and whiskers plots. Ct values are shown as median (lines), whereby the length of the box represents the $25^{\text {th }}$ and $75^{\text {th }}$ percentile, respectively. The symbol in the box indicates the mean. The vertical lines, i.e. whiskers, extend to the group minimum and maximum values. Upon subjective evaluation, $18 \mathrm{~S}$ showed the greatest degree of variation.

To evaluate expression stability, ratios of $\mathrm{Ct}$ values for all possible gene combinations were formed and graphed 
Table 2 Coefficient of variation of $\mathrm{Ct}$ values for transcripts tested in uterine, testicular, and conceptus tissue

\begin{tabular}{cccccc}
\hline \multicolumn{2}{c}{ Uterine tissue } & \multicolumn{2}{c}{ Testicular tissue } & \multicolumn{2}{c}{ Conceptus tissue } \\
\hline Transcript & CV (\%) & Transcript & CV (\%) & Transcript & CV (\%) \\
\hline GAPDH & 5.24 & GAPDH & 8.72 & 18S & 0.86 \\
ACTB & 6.39 & SDHA & 9.76 & GAPDH & 1.75 \\
B2M & 7.04 & GUSB & 9.89 & YWHZ & 1.87 \\
I8S & 17.07 & B2M & 10.67 & SDHA & 2.23 \\
& & ACTB & 12.37 & ACTB & 2.88 \\
& & 18S & 26.26 & B2M & 3.38 \\
\hline
\end{tabular}

in the form of box and whiskers plots (Figure 2). The gene comparisons involving $18 S$ rRNA showed the greatest extent of variability. This observation was also reflected in an increased $\mathrm{CV}$ for the Ct ratios when $18 \mathrm{~S}$ was compared with the other genes. Mean $\mathrm{Ct}$ ratios including $18 \mathrm{~S}$ had an average $\mathrm{CV}$ of 13.29 , whereas those ratios not including $18 \mathrm{~S}$ had an average $\mathrm{CV}$ of 4.37.

The stability values provided by NormFinder are listed in Table 3. B2M had the lowest stability value with 0.02 , thereby being the most stable gene among the tested transcripts. GAPDH and $A C T B$ had the same stability value of 0.028 , which was slightly higher than the value for $B 2 M .18 S$ was ranked the least stable gene with the highest stability value of 0.06 .

Upon analysis using geNorm, all four tested genes returned an average expression stability $M$ value of less than 1.5, indicating stable expression across samples (Figure 3). geNorm determined GAPDH and B2M to be the most stably expressed pair of genes.

To validate the above listed analysis concerning the stable expression of $18 S, G A P D H, A C T B$ and $B 2 M$, Ct values for $S L C 36 A 2$ were determined and normalized using each of the four putative reference genes. Normalization was carried out by determining $\Delta \mathrm{Ct}$ values for SLC36A2. Expression levels of SLC36A2 were then determined relative to levels observed at Day 8 of the estrous cycle by generating $\Delta \Delta \mathrm{Ct}$ values. Pearson's correlation analysis of these $\Delta \Delta \mathrm{Ct}$ values revealed a high degree of correlation. Each of the six possible pair wise correlations showed an $\mathrm{r}$-value of 0.99 and a p-value of less than 0.0001 . Average $\Delta \Delta \mathrm{Ct}$ values for each of the four putative reference genes and seven types of endometrial tissue are shown in Figure 4.

\section{Testis and associated structures}

$18 S$ showed the highest level of mRNA abundance with a mean Ct value of $8.19 \pm 0.39$, while the remaining transcripts showed similar abundance with a mean $\mathrm{Ct}$ value of $20.22 \pm 0.32(G A P D H), 20.67 \pm 0.4(B 2 M)$, $19.55 \pm 0.44$ (ACTB), $27.13 \pm 0.49$ (GUSB), and $23.65 \pm$ $0.42(S D H A)$ respectively.

Mean Ct values for $18 S$ displayed the greatest amount of deviation with a $\mathrm{CV}$ of $26.26 \%$. Mean Ct values for GAPDH displayed the least amount of variation with a $\mathrm{CV}$ of $8.72 \%$. The remaining transcripts showed a slightly higher CV than GAPDH (Table 2). Upon subjective evaluation of box and whiskers plots displaying the log transformed Ct values, $18 \mathrm{~S}$ showed the greatest degree of variation (Figure 1). Figure 2 shows box and whisker plots visualizing the mean ratios of all $\mathrm{Ct}$ values for each gene combination. Upon subjective evaluation the gene comparison involving $18 S \mathrm{rRNA}$ showed the greatest extent of variability, which was reflected in an increased CV for mean Ct ratios including 18S (15.75\% versus 5.61\% for Ct ratios not including $18 S$ ).

The stability values returned by NormFinder (Table 3) identified $B 2 M$ as the most stably expressed gene (0.018). ACTB and GAPDH had higher, i.e. less stable

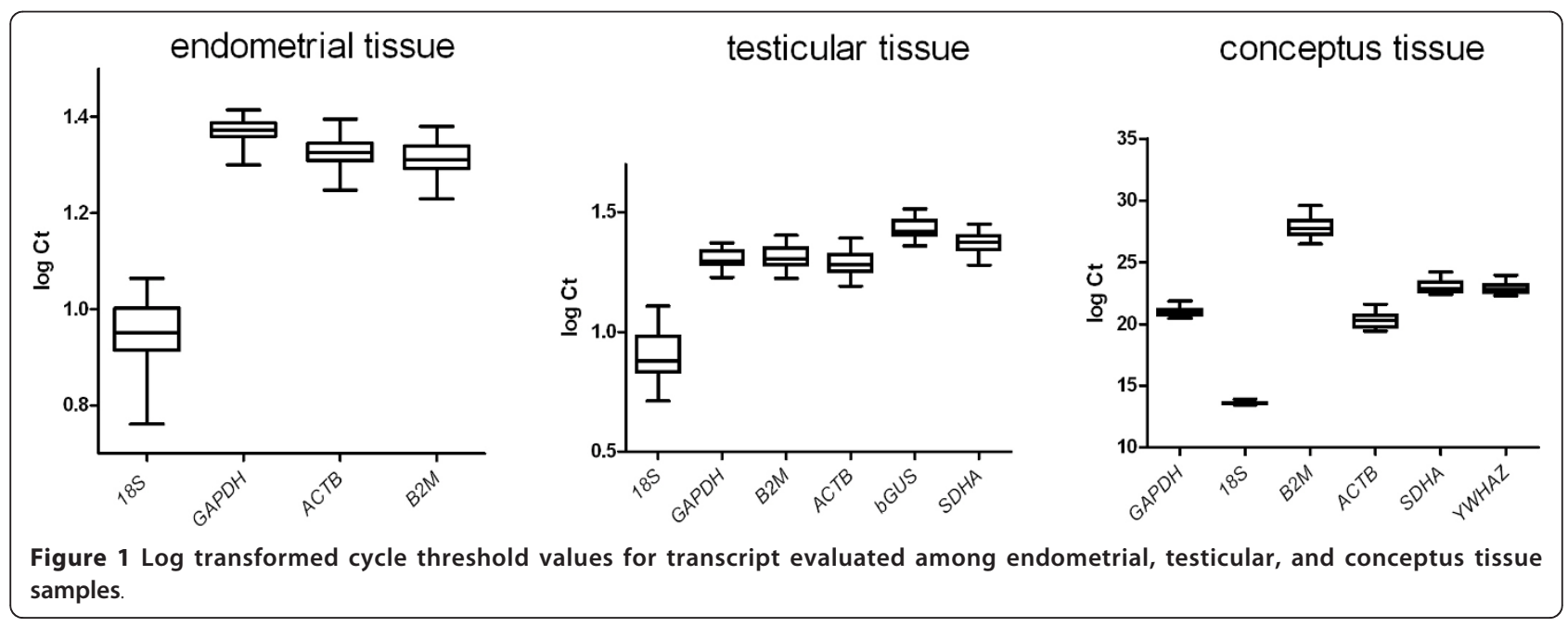




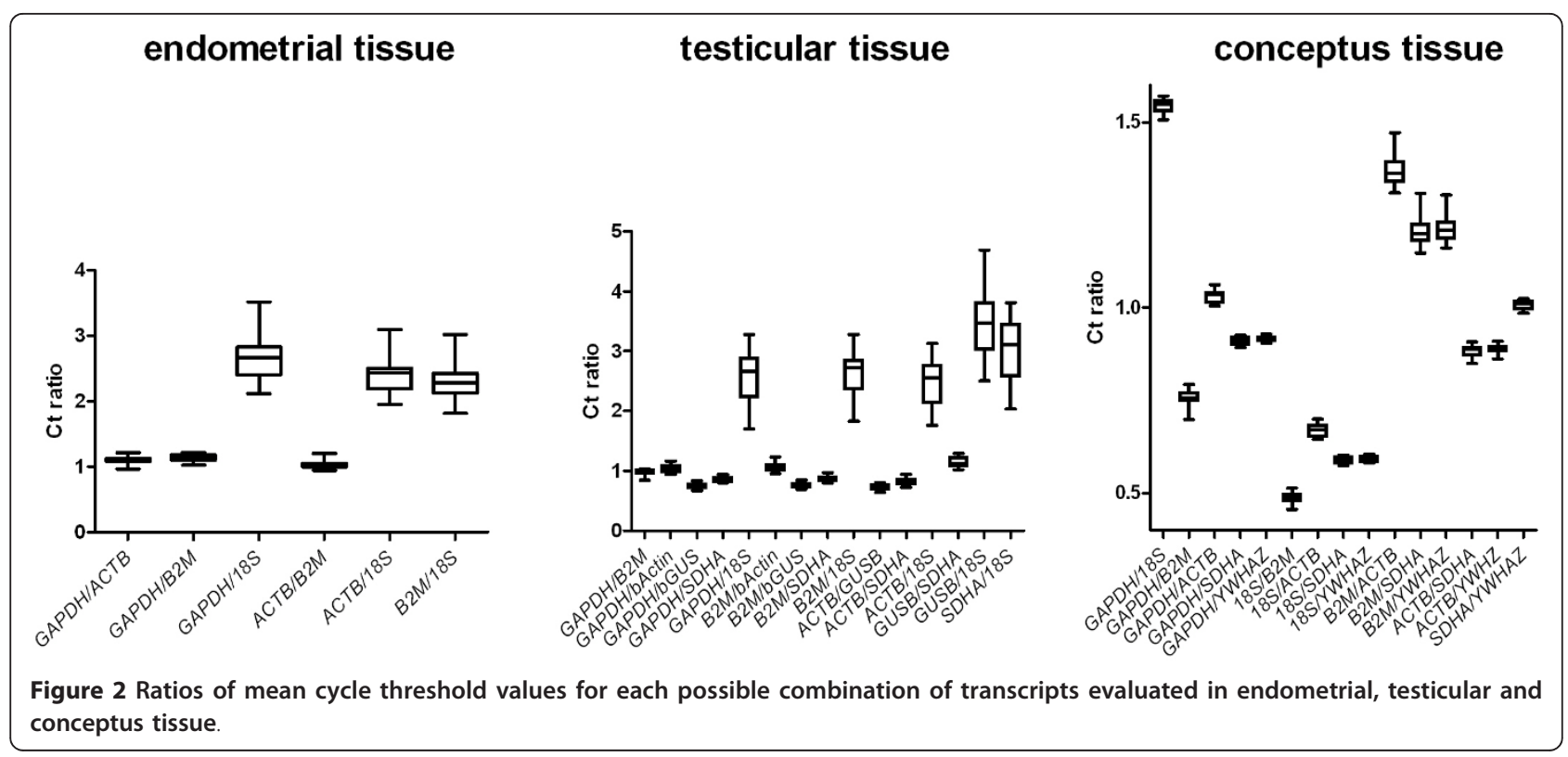

stability values than $B 2 M(0.033$ and 0.039$)$, followed by $S D H A$ and GUSB with a stability value of 0.043 each. $18 S$ was ranked the least stable transcript with a stability value of 0.163 .

Upon geNorm analysis, all six transcripts resulted in a $M$ value of less than 1.5, equating stable expression (Figure 5). geNorm furthermore determined $B 2 M$ and $18 S$ as the most stable gene combination.

To validate the above listed analyses concerning the stable expression of $18 S$, GAPDH, ACTB, B2M, SDHA, and GUSB, Ct values for Cyp19a1 were determined and normalized using each of the six putative reference genes. Normalization was carried out by determining $\Delta$ Ct values for Cyp19a1. Expression levels of Cyp19a1 were then determined relative to levels observed in testis by generating $\Delta \Delta \mathrm{Ct}$ values. Pearson's correlation analysis of these $\Delta \Delta \mathrm{Ct}$ values revealed a high degree of correlation. Each of the fourteen possible pair wise correlations showed an $\mathrm{r}$-value of 0.98 or higher and a $\mathrm{p}$-value of less than 0.0001 . Average $\Delta \Delta \mathrm{Ct}$ values for

Table 3 Gene stability values returned by Normfinder

\begin{tabular}{cccccc}
\hline \multicolumn{2}{c}{ Uterine tissue } & \multicolumn{2}{c}{ Testicular tissue } & \multicolumn{2}{c}{ Conceptus tissue } \\
\hline Transcript & $\begin{array}{c}\text { Stability } \\
\text { value }\end{array}$ & Transcript & $\begin{array}{c}\text { Stability } \\
\text { value }\end{array}$ & Transcript & $\begin{array}{c}\text { Stability } \\
\text { value }\end{array}$ \\
\hline B2M & 0.020 & B2M & 0.018 & YWHZ & 0.003 \\
GAPDH & 0.028 & ACTB & 0.033 & GAPDH & 0.007 \\
ACTB & 0.028 & GAPDH & 0.039 & SDHA & 0.010 \\
18S & 0.060 & SDHA & 0.043 & 18S & 0.013 \\
& & GUSB & 0.043 & ACTB & 0.015 \\
& & 18S & 0.163 & B2M & 0.029 \\
\hline
\end{tabular}

The lower the stability value, the more stable the gene is expressed. each of the four putative reference genes and four types testicular tissue are shown in Figure 6.

\section{Conceptus tissue}

$18 S$ showed the highest level of mRNA abundance with a mean Ct value of $13.58 \pm 0.03$, while $B 2 M$ exhibited the lowest levels with a mean $C t$ value of $27.81 \pm 0.24$. The remaining transcripts showed average expression levels with $20.97 \pm 0.09$ (GAPDH), $20.34 \pm 0.15$ (ACTB), $22.90 \pm 0.11(Y W H Z)$, and $23.06 \pm 0.13(S D H A)$.

Mean Ct values for $18 S$ displayed the least amount of deviation with a $\mathrm{CV}$ of $0.86 \%$. Mean $\mathrm{Ct}$ values for GAPDH displayed the greatest amount of variation with a $\mathrm{CV}$ of $3.38 \%$. Mean $\mathrm{Ct}$ values for remaining transcripts

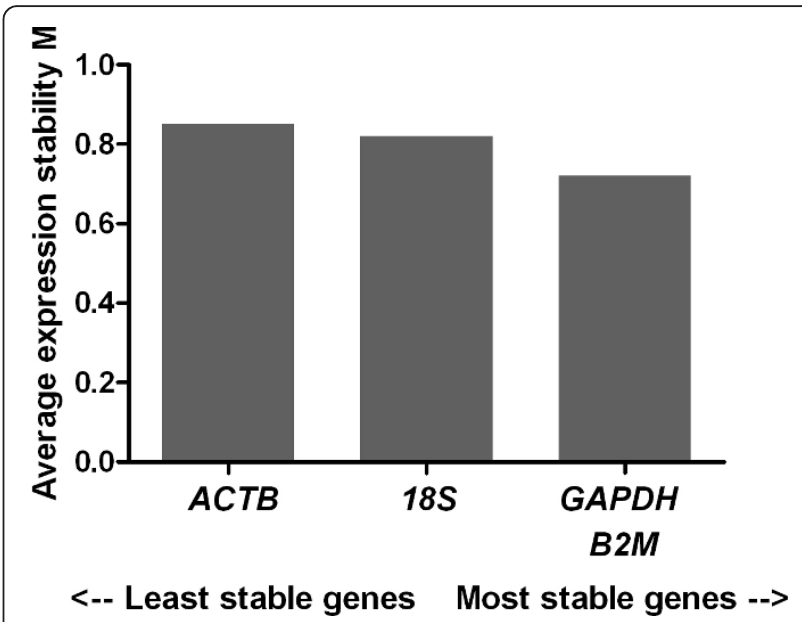

Figure 3 Expression stability values (M) of the putative reference genes tested in uterine tissue. Transcripts are ranked from the least stable to the most stable (left to right). 


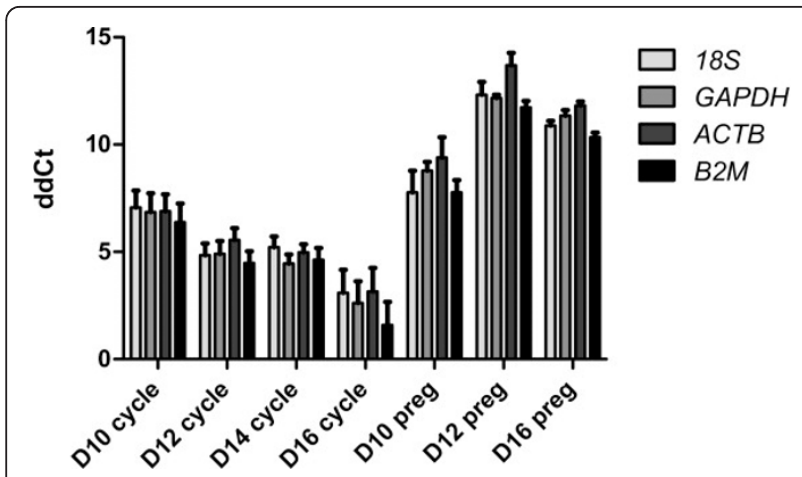

Figure 4 Relative quantification of SLC36A2 expression in uterine tissue. Displayed are average $\triangle \triangle C t$ values with SEM. $\triangle \Delta C t$ values generated using GAPDH for normalization showed the least amount of variability, with an average SEM of 1.06 , while $\Delta \Delta C t$ values generated using 185 for normalization showed the greatest degree of variability with an average SEM of 1.4.

showed as similar degree variation (Table 2). Figure 1 displays the log transformed mean Ct values for each of the four putative reference genes in the form of box and whiskers plots. Upon subjective evaluation $18 S$ showed the least, and $B 2 M$ the greatest degree of variation.

To evaluate expression stability, ratios of $\mathrm{Ct}$ values for all possible gene combinations were formed and graphed in the form of box and whiskers plots (Figure 2). The gene comparisons involving $B 2 M$ showed the greatest extent of variability. This observation was also reflected in an increased $\mathrm{CV}$ for the $\mathrm{Ct}$ ratios when $B 2 M$ was compared with the other genes. Mean Ct ratios including $B 2 M$ had an average CV of $13.29 \%$, whereas those ratios not including $B 2 M$ had an average $\mathrm{CV}$ of $4.37 \%$.

The stability values provided by NormFinder are listed in Table 3. YHWZ had the lowest stability value with 0.003 , thereby being the most stable gene among the

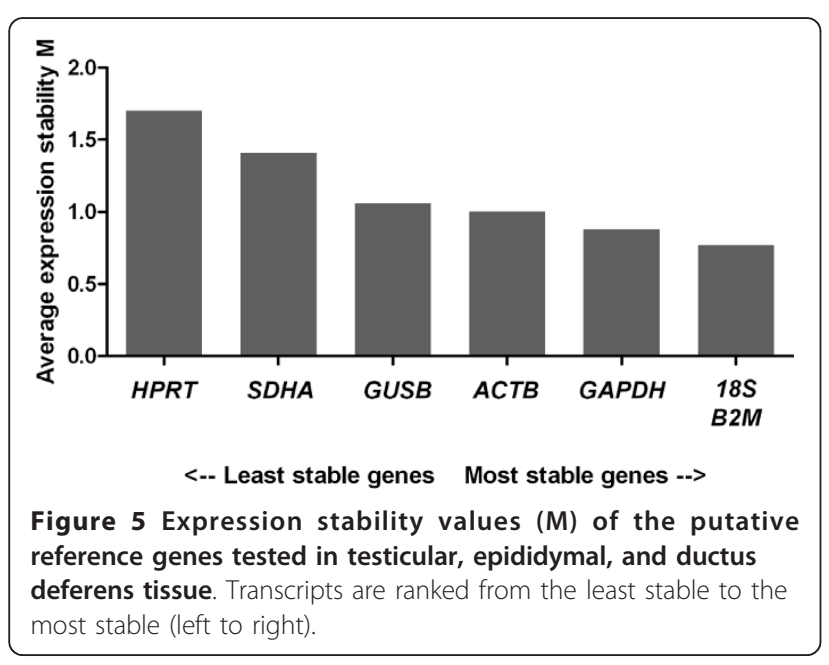

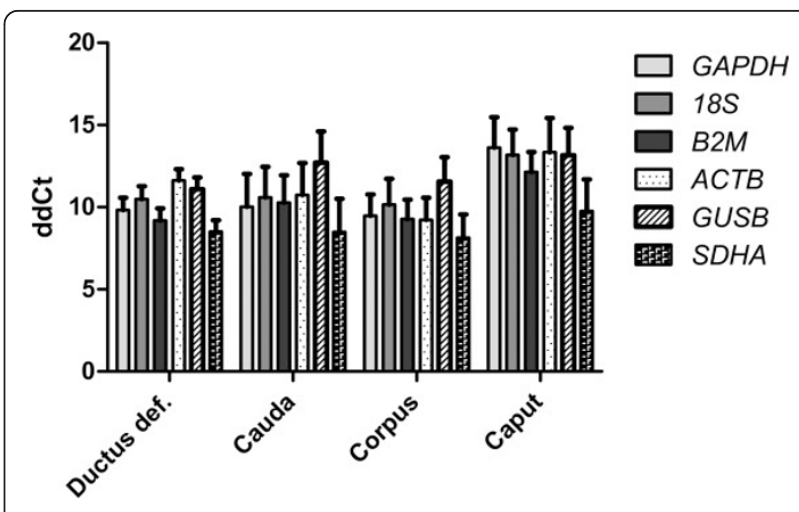

Figure 6 Relative quantification of Cyp19a1 expression in testicular, epididymal, and ductus deferens tissue. Displayed are average $\triangle \triangle C$ t values with SEM. $\triangle \Delta C$ t values generated using B2M for normalization showed the least amount of variability, with an average SEM of 1.2, while $\triangle \triangle C$ t values generated using SDHA and $A C T B$ for normalization showed the greatest degree of variability with an average SEM of 1.52 .

tested transcripts. $B 2 M$ was ranked the least stable gene with the highest stability value of 0.06 .

Upon analysis using geNorm, all six tested genes returned an average expression stability $M$ value of less than 1.5, indicating stable expression across samples (Figure 7). geNorm determined GAPDH and $Y H W Z$ to be the most stably expressed pair of genes, while $B 2 M$ was ranked least stable.

As described for uterine and testicular tissue, $\mathrm{Ct}$ values for Cyp19a1 were determined and normalized using each of the six putative reference genes. Normalization was carried out by determining $\Delta \mathrm{Ct}$ values for Cyp19a1. Expression levels of Cyp19a1 were then determined relative to levels observed in Day 8 conceptuses by generating $\Delta \Delta \mathrm{Ct}$ values. Pearson's correlation analysis of these $\Delta \Delta \mathrm{Ct}$ values revealed a high degree of correlation. Pair wise correlations no including B2M showed an $\mathrm{r}$-value of 0.98 or greater and a p-value of less than 0.0001 . Pair wise correlations including $B 2 M$ showed an

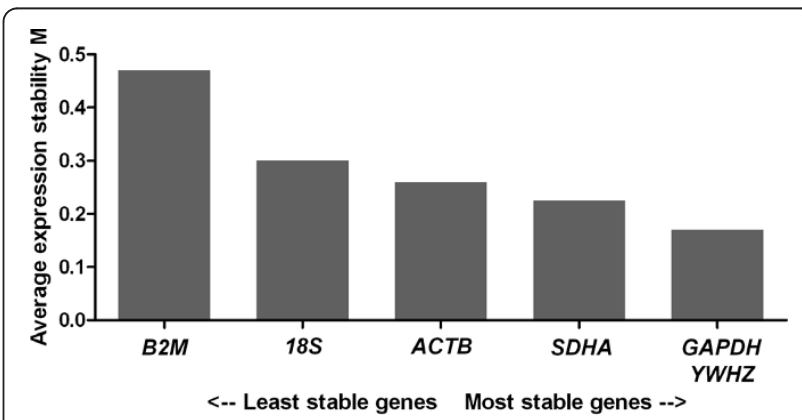

Figure 7 Expression stability values $(M)$ of the putative reference genes tested in conceptuses. Transcripts are ranked from the least stable to the most stable (left to right). 
average r-value of 0.83 and a p-value of 0.001 . Average $\Delta \Delta \mathrm{Ct}$ values for each of the six putative reference genes and three developmental stages of conceptus development are shown in Figure 8.

\section{Discussion}

Real time RT-PCR is one of the most sensitive and reproducible quantification methods for gene expression analysis. Selection of internal control genes is a crucial step in the experimental design process, as relative quantification procedures rely on the stable expression of such internal control or reference genes among samples regardless of the biological conditions. The most suitable reference gene has to be determined for each tissue as a gene stably expressed in one type of tissue is not necessarily stably expressed in another type of tissue. However, control genes are commonly chosen on the basis of observations from research on other tissue types or on the same tissue type in a different species. The present study was performed to determine the suitability of selected putative internal control genes in three different types of tissues when performing realtime RT-PCR analysis in the equine species.

\section{Uterine samples}

$18 S$ rRNA, GAPDH, $A C T B$, and $B 2 M$ are commonly used as internal control genes when performing quantitative PCR analysis on uterine tissue samples derived from species such as human or mouse. As expected, $18 \mathrm{~S}$ $r R N A$ showed the highest level of mRNA abundance while $G A P D H, A C T B$, and $B 2 M$ showed lower expression levels. The average cycle threshold value across all 35 analyzed samples showed the greatest degree of variability for $18 S$ rRNA whereas the remaining three transcripts displayed a low degree of variation. Ct ratios for all possible pair wise combinations were formed. Assuming stable expression, one would expect a low

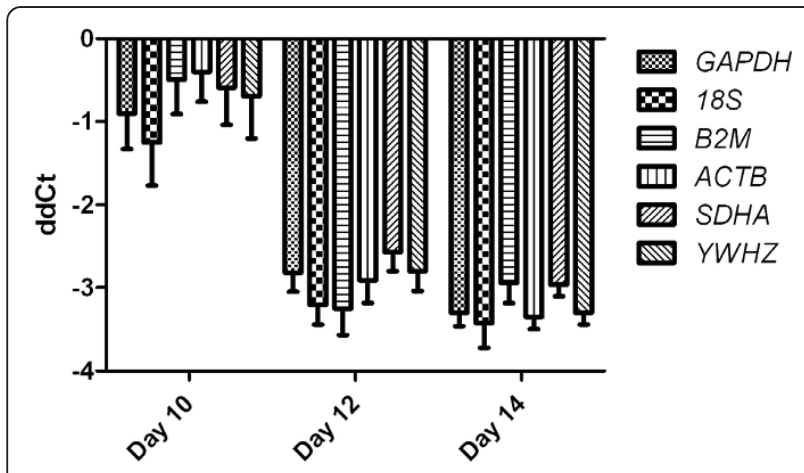

Figure 8 Relative quantification of Cyp19a1 expression in conceptuses. Displayed are average $\triangle \triangle C$ t values with SEM. $\Delta \Delta C t$ values generated showed similar degree of variability for all transcripts analyzed. variability in the resulting ratios. However, a pattern emerged, whereby comparisons including $18 S$ displayed increased deviation, and comparisons excluding $18 \mathrm{~S}$ showed low deviation. This is in accordance with the greater degree of variability observed for $18 \mathrm{~S}$ Ct values. NormFinder, a publicly available program that ranks transcripts according to their expression stability, returned $18 S$ as the least stably expressed gene supporting the results of the above mentioned descriptive analyses. $B 2 M$ was listed the most stable expressed gene, closely followed by GAPDH and $A C T B$. geNorm identified $G A P D H$ and $B 2 M$ as the most stably expressed pair of genes. Overall, all four genes were identified as stable amongst the samples analyzed with $18 S$ being slightly less stably expressed than GAPDH, B2M, and ACTB.

To validate the suitability of the tested transcripts as reference genes, expression levels for SLC36A2 were determined. SLC36A2 had previously been identified as highly up-regulated during early pregnancy and was chosen with the hypothesis that its expression levels would vary throughout the estrous cycle and early pregnancy [8]. Cycle threshold values for SLC36A2 were normalized using each of the four putative reference genes through generating $\Delta \mathrm{Ct}$ values. Expression values relative to levels observed at Day 8 of the estrous cycle were determined through determining $\Delta \Delta \mathrm{Ct}$ values. Pair wise comparisons of resulting $\Delta \Delta \mathrm{Ct}$ values revealed that all six possible pair wise combinations were highly correlated. High correlation of $\Delta \Delta \mathrm{Ct}$ values reflects the similar expression of all putative reference genes across the tested samples. Average $\Delta \Delta \mathrm{Ct}$ values for each of the seven biological conditions showed the greatest degree of variation when $18 S$ was used for normalization, while GAPDH as normalization factor resulted in the least degree of variability. This finding supports the above stated conclusion that all four genes were identified as stably amongst the samples analyzed with $18 \mathrm{~S}$ being slightly less stably expressed than GAPDH, B2M, and ACTB.

Surprisingly few studies concerning the ideal selection of reference genes in endometrial tissue have been published. Murray and others [9] evaluated the appropriateness of several transcripts as reference genes on endometrial tissue specimens obtained from women throughout the menstrual cycle and then tested the suitability of these genes to detect the variation of estrogen and progesterone receptor levels during the course of the cycle. geNorm determined GAPDH to be amongst the most stably expressed genes, which is in agreement with the results of the current study in horse tissues. $A C T B$, which expression was ranked as stable according to geNorm, however failed to detect changes in endometrial estrogen and progesterone receptor levels and was therefore determined to be not suitable as a 
reference gene. This underlines the importance to not only test the stability of a putative reference gene for a specific tissue type, but also the necessity to evaluate each species individually. Walker and co-workers evaluated a set of endogenous control genes for bovine endometrial tissue. The transcripts tested in that study however did not overlap with those tested in the present study so that a comparison of results is not possible [10]. The expression of $18 S$, the transcript showing the greatest variation across the 35 samples analyzed in this study, has been identified as regulated by progesterone in the murine uterus [11]. This observation may provide some explanation for the observed variation of $18 \mathrm{~S}$ in equine endometrial tissue samples collected throughout the estrous cycle, as these samples have been exposed to endogenous progesterone of luteal origin for variable time periods.

\section{Testis and associated structures samples}

Gene expression levels of $18 S$, GAPDH, B2M, ACTB, $G U S B$, and $S D H A$ were measured in 30 tissue samples collected from testis, epididymis, and ductus deferens. As expected, $18 S$ rRNA showed the highest level of mRNA abundance. The average cycle threshold value across all samples analyzed showed the greatest degree of variability for $18 S$ rRNA whereas the remaining four transcripts displayed a low, and GAPDH the lowest degree of variation. $C t$ ratios for all possible pair wise combinations were formed, whereby comparisons including $18 \mathrm{~S}$ displayed increased deviation, and comparisons excluding $18 S$ showed low deviation. This is in accordance with the greater degree of variability observed for $18 \mathrm{~S} \mathrm{Ct}$ values. NormFinder identified $18 \mathrm{~S}$ as the least stably expressed gene supporting the results of the above mentioned descriptive analyses. Upon geNorm analysis, $18 S, B 2 M$ and GAPDH were listed as the most stably expressed genes. Surprisingly, geNorm identified $B 2 M$ and $18 S$ as the most stably expressed pair of genes. This finding disagrees with NormFinder, which ranked $18 S$ as the least stable transcript and in addition does not match the descriptive analysis of the dataset which identified $18 S$ as the gene with the largest degree of variability. This is the only case throughout this data analysis in which results returned by NormFinder and geNorm do not match. The descriptive analysis however supports the results returned by NormFinder.

As performed for the analysis on uterine tissue, the suitability of the tested transcripts as reference genes was validated through determining the relative expression levels for a non-reference transcript, in this case the relative expression of Cyp19a1 to levels observed in testis. Cyp19a1, an enzyme involved in the biosynthesis of estrogens was chosen because of its known high level of expression in testicular tissue. Pair wise comparisons of resulting $\Delta \Delta \mathrm{Ct}$ values revealed that all thirteen possible pair wise combinations were highly correlated.

The authors were not able to find information regarding the evaluation of internal control genes in testicular, epididymal, or ductus deferens tissue, neither in the horse nor in any other species. Overall, the current analysis revealed $B 2 M$ and $G A P D H$ as the most stable expressed transcripts in the set of tissue used and are therefore recommended to use as internal control genes.

\section{Conceptuses}

As seen for uterine and testicular tissue, $18 S$ showed the highest mRNA abundance. B2M exhibited lowest mRNA levels with the remaining transcripts at intermediate levels. Unlike the other types of tissues tested $18 S$ mRNA showed the least amount of variation among the transcripts analyzed with a $\mathrm{CV}$ of mean $\mathrm{Ct}$ values of less than one percent. $B 2 M$ showed the greatest degree of variability during the course of the descriptive analysis which became especially evident when forming ratios of $\mathrm{Ct}$ values for all possible gene combinations. Likewise, $B 2 M$ was identified as the least stable expressed gene by NormFinder and geNorm, while $Y W H Z$ and GAPDH were determined to be the most stable transcripts. Despite the descriptive analysis, which revealed $18 S$ as the transcript with the least amount of variation, i.e. the most stable gene, both NormFinder and geNorm did not rank $18 S$ as the most stably expressed gene. Cyp19a1 mRNA levels were employed to determine relative expression levels for a non-reference transcript, as abundance for this transcript had previously been identified to change with conceptus development (Klein, unpublished observation). Formation of $\Delta \Delta \mathrm{Ct}$ values relative to Day 8 conceptuses for Cyp19a1 and their pair wise comparisons revealed high correlation for combinations not including $B 2 M$ normalized $\Delta \Delta \mathrm{Ct}$ values, further corroborating the above listed findings.

Overall, the six reference genes tested in conceptus' tissue showed less variability in expression than the reference genes tested in uterine and testicular samples. Pre-implantation conceptuses are less complex in their composition than uterine and testicular tissues, most likely contributing to the decreased variability. Smits and co-workers [12] determined the expression of 8 candidate reference genes in equine blastocysts which is a stage of embryonic development equivalent to the Day 8 conceptuses in the current study. According to the Mvalue returned by geNorm, transcripts analyzed in that study were less stably expressed than in the current study. In the present study, cDNA was quantified after reverse transcription, thereby providing more control over the amount of cDNA used as input for each RTqPCR reaction. The finding by Smits et al. that $S D H A$ appeared to be highly regulated in equine blastocysts, 
could not be confirmed and is in line with the stable expression of SDHA described for bovine embryos [13].

In general the current analysis revealed $18 S, G A P D H$, and $Y W H Z$ to be the most stable transcript in preimplantation equine embryos and are recommended as reference genes for RT-qPCR experiments.

\section{Author details}

${ }^{1}$ University of Kentucky, Department of Veterinary Science, 108 Gluck Equine Research Center, Lexington, KY, 40546, USA. ${ }^{2}$ College of Veterinary Medicine, Western University of Health Sciences, 309 East Second Street, Pomona, CA 91766, USA.

\section{Authors' contributions}

CK designed and conducted the study. JR participated in design of the study and drafting the manuscript. MHT conceived the study, participated in its coordination and helped to draft the manuscript. All authors read and approved the final manuscript.

\section{Competing interests}

None of the authors in this study has any financial personal relationship with any organization that could influence (bias) this work.

Received: 2 October 2010 Accepted: 12 April 2011

Published: 12 April 2011

\section{References}

1. Ferre F: Quantitative or semi-quantitative PCR: reality versus myth. $P C R$ Methods Appl 1992, 2:1-9.

2. Bogaert L, Van Poucke M, De Baere C, Peelman L, Gasthuys F, Martens A: Selection of a set of reliable reference genes for quantitative real-time PCR in normal equine skin and in equine sarcoids. BMC Biotechnol 2006, 6:24.

3. Cappelli K, Felicetti M, Capomaccio S, Spinsanti G, Silvestrelli M, Supplizi AV: Exercise induced stress in horses: selection of the most stable reference genes for quantitative RT-PCR normalization. BMC Mol Biol 2008, 9:49.

4. Bustin SA, Benes V, Garson JA, Hellemans J, Huggett J, Kubista M, Mueller R, Nolan T, Pfaffl MW, Shipley GL, et al: The MIQE guidelines: minimum information for publication of quantitative real-time PCR experiments. Clin Chem 2009, 55:611-622.

5. Pfaffl MW, Tichopad A, Prgomet C, Neuvians TP: Determination of stable housekeeping genes, differentially regulated target genes and sample integrity: BestKeeper-Excel-based tool using pair-wise correlations. Biotechnol Lett 2004, 26:509-515.

6. Andersen $\mathrm{CL}$, Jensen $\mathrm{J}$, Orntoft TF: Normalization of real-time quantitative reverse transcription-PCR data: a model-based variance estimation approach to identify genes suited for normalization, applied to bladder and colon cancer data sets. Cancer Res 2004, 64:5245-5250.

7. Vandesompele J, De Preter K, Pattyn F, Poppe B, Van Roy N, De Paepe A, Speleman F: Accurate normalization of real-time quantitative RT-PCR data by geometric averaging of multiple internal control genes. Genome Biol 2002, 3:RESEARCH0034.

8. Klein C, Scoggin KE, Ealy AD, Troedsson MH: Transcriptional Profiling of Equine Endometrium During the Time of Maternal Recognition of Pregnancy. Biol Reprod 2010.

9. Murray RS, Inman DA, Yuan L, Fritz MA, Young SL: Choice of a constitutive housekeeping gene (HKG) is critical in the analysis of real-time reverse transcriptase polymerase chain reaction (qRT-PCR) results. Fertil Steril 2010, 86(3):S38-S39.

10. Walker CG, Meier S, Mitchell MD, Roche JR, Littlejohn M: Evaluation of realtime PCR endogenous control genes for analysis of gene expression in bovine endometrium. BMC Mol Biol 2009, 10:100.

11. Craythorn RG, Girling JE, Hedger MP, Rogers PA, Winnall WR: An RNA spiking method demonstrates that 18S rRNA is regulated by progesterone in the mouse uterus. Mol Hum Reprod 2009, 15:757-761.

12. Smits K, Goossens K, Van Soom A, Govaere J, Hoogewijs M, Vanhaesebrouck E, Galli C, Colleoni S, Vandesompele J, Peelman L: Selection of reference genes for quantitative real-time PCR in equine in vivo and fresh and frozen-thawed in vitro blastocysts. BMC Res Notes 2009, 2:246.

13. Goossens K, Van Poucke M, Van Soom A, Vandesompele J, Van Zeveren A Peelman LJ: Selection of reference genes for quantitative real-time PCR in bovine preimplantation embryos. BMC Dev Biol 2005, 5:27.

doi:10.1186/1756-0500-4-120

Cite this article as: Klein et al:: Expression stability of putative reference genes in equine endometrial, testicular, and conceptus tissues. BMC Research Notes 2011 4:120.

\section{Submit your next manuscript to BioMed Central and take full advantage of:}

- Convenient online submission

- Thorough peer review

- No space constraints or color figure charges

- Immediate publication on acceptance

- Inclusion in PubMed, CAS, Scopus and Google Scholar

- Research which is freely available for redistribution 